\title{
An effective molecular blockers of ion channel of M2 protein as anti-influenza A drug
}

\author{
Yury Nikolaevich Vorobjev \\ Institute of Chemical Biology and Fundamental Medicine, Siberian Branch of Russian Academy of Sciences, Novosibirsk 630090, Russia \\ ynvorob@niboch.nsc.ru
}

Key words - Influenza A; M2 protein; ion channel blockers

\section{Motivation:}

Design of a drug molecule that can effectively bind to the M2 ion channel and block a diffusion of ions $\mathrm{H}^{+}$through and inhibit influenza A virus replication is an important task.

\section{Methods}

A new class of positively charged, +2 , molecules is proposed to block diffusion of $\mathrm{H}^{+}$ion through the $\mathrm{M} 2$ channel. Several drug candidates, derivatives of a lead compound (diazabicyclooctane), is proposed and investigated. Molecular dynamics of thermal fluctuations of M2 protein structure and ionization-conformation coupling of all the ionizable residues were simulated at physiological $\mathrm{pH}$ via original methods [1]. The influence of the most probable mutations of key drugbinding amino acid residues in the M2 ion channel are investigated too.

\section{Results}

It is shown that the suggested blocker drug molecule has high binding affinity for the native and mutant M2 ion channel. There are two in-channel binding sites of high affinity for the native M2 protein, the first one demonstrates formation of two H-bonds with two of four serine residues Ser-31A (B) or Ser-31C(D), and the second one has H-bonds with two of four histidine residues His-37A (B), or His$37 \mathrm{C}(\mathrm{D})$. Six types of the most probable mutations of residues Ser-31A(B,C,D) are analyzed by the same computation protocol, as for the native M2 protein, and it is shown that the binding site with His-37A(B,C,D) residues is highly conservative with high binding affinity. Probability of double mutants, namely Ser-32 and His-37 is quite low and does not exceed $10^{-5}$. The main advantages of the new drug molecule is the positive charge, +2 , which creates a positive electrostatic potential barrier (in addition to a steric one) for a transfer of $\mathrm{H}^{+}$ion through M2 ion channel and may serve as an effective anti-influenza A virus drug.

\section{ACKNOWLEDGEMENTS}

This research was supported by the Russian Foundation for Basic Research (№ 18-04-00005-a) and a RussianGovernment-funded budget project for the Institute of Chemical Biology and Fundamental Medicine, the Siberian Branch of the Russian Academy of Sciences: № AAAA-A17117020210022-4.

\section{REFERENCES}

[1] Vorobjev, Y.N. Modeling of Electrostatic Effectsin Macromolecules, 163-202. Computational Methods to Study the Structure and Dynamics of Biomolecules. Springer Nature, 2019. ISSN 978-3-319-95843-9; https://doi.org/10.1007/978-3-319-95843-9_6 